\title{
Design of an Optimal Bayesian Incentive Compatible Broadcast Protocol for Ad Hoc Networks with Rational Nodes
}

\author{
N. Rama Suri and Y. Narahari
}

\begin{abstract}
Nodes in an ad hoc wireless network incur certain costs for forwarding packets since packet forwarding consumes the resources of the nodes. If the nodes are rational, free packet forwarding by the nodes cannot be taken for granted and incentive based protocols are required to stimulate cooperation among the nodes. Existing incentive based approaches are based on the VCG (Vickrey-Clarke-Groves) mechanism which leads to high levels of incentive budgets and restricted applicability to only certain topologies of networks. Moreover, the existing approaches have only focused on unicast and multicast. Motivated by this, we propose an incentive based broadcast protocol that satisfies Bayesian incentive compatibility and minimizes the incentive budgets required by the individual nodes. The proposed protocol, which we call $B I C$-B (Bayesian incentive compatible broadcast) protocol, also satisfies budget balance. We also derive a necessary and sufficient condition for the ex-post individual rationality of the BIC-B protocol. The BIC-B protocol exhibits superior performance in comparison to a dominant strategy incentive compatible broadcast protocol.
\end{abstract}

Index Terms-Ad hoc wireless networks, incentive compatible broadcast (ICB), rationality, selfish nodes, Bayesian incentive compatible broadcast (BIC-B), Dominant strategy incentive compatible broadcast (DSIC-B), least cost path (LCP), source rooted broadcast tree (SRBT), budget balance, individual rationality.

\section{INTRODUCTION}

W IRELESS communications industry is currently one of the fastest growing industries in the world. The industry has several segments such as cellular telephony, satellite-based communication networks, wireless LANs, and ad hoc wireless networks. An ad hoc wireless network is an autonomous system of nodes connected through wireless links. It does not have any fixed infrastructure such as base stations in cellular networks. The nodes in the network coordinate among themselves for communication. Hence, each node in the network, apart from being a source or destination, is also expected to route packets for other nodes in the network. Such networks find varied applications in real-life environments such as communication in battle fields, communication among rescue personnel in disaster affected areas, and wireless sensor networks.

The conventional protocols for routing, multicasting, and broadcasting in ad hoc wireless networks assume that nodes

Manuscript received August 1, 2007; revised March 10, 2008. The research is supported by ONR Grant No. N0014-06-1-0994.

Y. Narahari is a Professor in the Department of Computer Science and Automation, Indian Institute of Science, Bangalore, India (e-mail: hari@csa.iisc.ernet.in).

N. Rama Suri is a doctoral student in the Department of Computer Science and Automation, Indian Institute of Science, Bangalore (e-mail: nrsuri@csa.iisc.ernet.in).

Digital Object Identifier 10.1109/JSAC.2008.080911. follow the prescribed protocol without any deviation and they cooperate with one another in performing network functions such as packet forwarding, etc. However, in many current applications of ad hoc wireless networks, nodes are autonomous, rational, and intelligent, and could exhibit strategic behavior. A wireless node is autonomous because no other wireless node may have direct control over the decisions or actions taken by that node. A wireless node is rational in the sense of making decisions consistently in pursuit of its own objectives. Each node's objective is to maximize the expected value of individual payoff measured in some utility scale. Note that selfishness or self-interest is an important implication of rationality. A wireless node is intelligent in the sense that it takes into account its knowledge or expectation of behavior of other nodes in determining its best response actions. The behavior exhibited by a network of rational and intelligent nodes can be modeled in a natural way using game theory [1]. We use the phrase selfish nodes in this paper, to refer to rational and intelligent nodes.

There are several recent efforts exploring the use of game theoretic models in the modeling and analysis of various design problems in ad hoc networks at different levels of the protocol stack [2], [3]. It has been applied at (a) the physical layer level to analyze distributed power control [4], [5] and waveform adaption; (b) at the data link layer level to analyze medium access control [6] and the reservation of bandwidth [7], [8]; and (c) at the network layer level to model the behavior of the packet forwarding strategies [9], [10]. Applications at the transport layer and above also exist, although less pervasive in the literature.

A question of interest in all the situations mentioned above is that of how to provide appropriate incentives to prevent selfish behavior by the nodes. Examples of selfish nodes include: a node increasing its power without regard for interference it may cause on its neighbors; a node immediately retransmitting a frame in the case of a collision without waiting for the backoff phrase; a node refusing to forward the transit packets of the other nodes in the network, etc. Selfish behavior is generally detrimental to the overall network performance. In this paper, our focus is on studying the packet forwarding strategies of selfish nodes in the specific context of broadcast.

\section{A. The Incentive Compatible Broadcast (ICB) Problem}

Broadcast is useful in ad hoc wireless networks in many situations, for example, route discovery, paging a particular host, or sending an alarm signal, etc. Successful broadcast requires appropriate forwarding of the packet(s) by individual 
wireless nodes. The nodes incur certain costs for forwarding packets since packet forwarding consumes the resources of the nodes. If the nodes are rational, we cannot take packet forwarding by nodes for granted. Reimbursing the forwarding costs or transit costs incurred by the nodes is a way to make them forward the packets. For this, we need to know the exact transit costs at the nodes. However, the nodes may not be willing to reveal the true transit costs. Broadcast protocols that induce revelation of true transit costs by the individual wireless nodes can be called incentive compatible, following mechanism design terminology. We can design an incentive compatible broadcast protocol by prescribing an appropriate allocation rule and payment rule into the broadcast protocol. We shall refer to the problem of designing such robust broadcast protocols as the incentive compatible broadcast (ICB) problem [11]. In this paper, we address the ICB problem in ad hoc wireless networks and offer an elegant solution using mechanism design theory.

\section{B. Relevant Work}

In the recent times, routing in the presence of selfish nodes has received significant attention, driven by the need to design protocols, like routing protocols, multi-cast protocols, etc., for networks with selfish nodes.

The early research in cooperation of stimulation in ad hoc wireless networks used a reputation mechanism [12]. These approaches use techniques such as auditing, use of appropriate hardware, system-wide optimal point analysis to identify selfish nodes and isolate non-cooperative nodes from the network. The watchdog-mechanism in Marti, Giuli, Lai, Baker [13], the core mechanism in Michiardi, and Molva [14], the confidant mechanism in Buchegger, and Boudec [15], etc. are a few examples of reputation mechanisms. These methods look for a system-wide optimum point which may not be individually optimal. Also, the use of hardware is not always feasible in network settings.

Srinivasan, Nuggehalli, and Chiasserini [16] modeled the routing situation using the repeated prisoner's dilemma problem. According to evolutionary game theory, an effective strategy in this kind of setting is the so-called TIT-FOR-TAT strategy. In Altman, Kherani, Michiardi and Molva [17], each node is assumed to forward packets with some probability which is independent of the source. These models do not take the dynamics of the network into consideration. A game theoretic model was introduced by Urpi, Bonuccelli and Giordano [18] based on static Bayesian games [1] to model forwarding behavior of selfish nodes in ad hoc wireless networks. Although this model properly formulates the game that the nodes are playing, it does not allow non-simultaneous decision making. In addition, the strategies in this framework are not dependent on past behavior. To get around these problems, Nurmi [19] modeled routing in ad hoc wireless networks with selfish nodes as a dynamic Bayesian game. This model is rich in the sense that it allows non-simultaneous decision making and incorporating history information into the decision making process.

Another important approach to designing incentive mechanisms is based on the techniques of microeconomics. Mechanism design is a powerful tool to model such situations. There have been several efforts to design incentive mechanisms for routing in ad hoc wireless networks in the presence of selfish nodes. Feigenbaum, Papadimitriou, Sami, and Shenker [20] and Hershberger and Suri [21] developed an incentive mechanism to address the truthful low cost routing (unicast) problem. The model consists of $n$ nodes where each node represents an autonomous system. They assume that each node $k$ incurs a transit $\operatorname{cost} c_{k}$ for forwarding one transit packet. For any pair of nodes $i$ and $j$ of the network, $T_{i, j}$ is the total amount of traffic originating from $i$ and destined for node $j$. The payments to nodes are computed using the VickreyClarke-Groves (VCG) payment rules [22], [23]. The authors in [20] and [21] presented a distributed method such that each node $i$ can compute its payment $p_{i, j}^{l}$ to node $l$ for carrying the transit traffic from node $i$ to node $j$ if node $l$ is on the least cost path from $i$ to $j$.

A similar type of model was presented by Anderegg and Eidenbenz [9] for stimulating cooperation among selfish nodes in ad hoc networks using an incentive scheme. This model generalizes one aspect of [20] by associating several costs to each node, one per each neighbor, instead of just one. This leads to a model based on edge weighted graph representation of the ad hoc network. The VCG mechanism is used to compute a power efficient path, where each node determines the power level required to transit/forward the packets. A node by itself cannot determine its power level required because it needs feedback information in the form of packets from its neighbors. As the nodes are selfish and non-cooperative, this feedback information may allow a node to cheat its neighbors in order to raise its own payoff. The authors of [9] did not address this issue. Eidenbenz, Santi, and Resta [24] modified the model in Anderegg and Eidenbenz [9] by using the VCG mechanism to compute the payments to the nodes, but the sender is charged the total declared cost of a second least cost path, that is the least cost path with all nodes in the cost efficient path removed. This requires the existence of at least two node disjoint paths between the sender and the receiver. Wang and $\mathrm{Li}$ [25] proposed strategy-proof mechanisms for the truthful unicast problem. They also presented an algorithm for fast computation of payments to nodes and a distributed algorithm for payment computation.

Zhong, Li, Liu, and Yang [26] used a two-stage routing protocol to model the routing problem in ad hoc wireless networks with selfish nodes. They integrated a novel cryptographic technique into the VCG mechanism to solve the link cost dependence problem. Zhong, Chen, and Yang [27] proposed a system called Sprite, which combines incentive methods and cryptography techniques to implement a group cheat-proof ad hoc routing system. Lu, Li, Wu [28] embed an incentivecompatible, efficient, and individual rational payment scheme into the routing protocol in ad hoc networks which consist of selfish nodes. Unlike traditional routing protocols in ad hoc networks, which only elicit cost information from selfish nodes, this model motivates selfish nodes to report truthfully both their stability and cost information.

In all the above solution approaches for the incentive compatible unicast problem (also known as the truthful unicast problem), the intermediate nodes on the path between the source node and the destination node are compensated for 


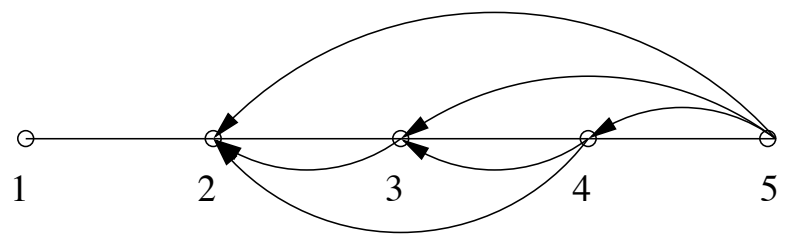

Fig. 1. Relating incentive compatible unicast solution to the $I C B$ problem

forwarding the packet. Let us apply this technique of service reimbursement to the incentive compatible broadcast (ICB) problem and see the consequences. Consider a portion of the network as shown in Figure 1. Let us consider node 1 to be the source of broadcast. Nodes 2, 3, 4, and 5 are the intended destinations of the broadcast packet. Node 5 needs to reimburse the nodes 2,3 , and 4 , since these nodes are intermediate nodes on the path between node 1 and node 5 and they forward the broadcast packet. For similar reasons, node 4 needs to reimburse the nodes 2 and 3 and node 3 needs to reimburse node 2 . On the whole, node 2 receives payments from nodes 3,4 , and 5 for forwarding a packet once. Similarly, node 3 receives payments from node 4 , and node 5 . Hence we end up with high values of over payments leading to an inefficient solution to the $I C B$ problem. It is important to observe, in the context of broadcast, that all the nodes in the network are intended destinations and there is no concept of intermediate nodes as in the case of unicast.

Wang and Li [29], [10] have proposed strategy-proof mechanisms for the truthful multicast problem in ad hoc wireless networks with selfish nodes. The authors, in this model, assume that the nodes in the multicast set forward the packets for free among themselves. However, this assumption may not be credible in real world applications, especially when the nodes are rational. In our work, we assume that nodes, being rational, do not necessarily forward the broadcast packets for free. This assumption clearly captures the real-world more accurately.

Suri and Narahari [30], [31] proposed a dominant strategy incentive compatible mechanism, DSIC-B, that is built into the corresponding broadcast protocol, as a solution to the ICB problem. In the DSIC-B protocol, a node pays only for the node from which it has received the broadcast packet and these payments are designed such that the mechanism is truthful in the dominant strategy sense, that is, true cost revelation is a best response for each node irrespective of what the other nodes report. There are, however, two limitations of using the DSIC-B protocol [30], [31].

- (L1): The underlying graph of ad hoc wireless network must be bi-connected.

- (L2): The DSIC-B protocol is not budget balanced [22], [32].

The limitation (L1) implies that the DSIC-B protocol works only for networks where the underlying graph of that network is bi-connected. This means that there cannot exist any cut vertex in the underlying graph. As the number of nodes in the network increases, sustaining this condition is difficult. The limitation (L2) implies that certain external budget needs to be injected into the network to sustain the running of the protocol.

To summarize the relevant work,
- Several game theoretic models and mechanism design solutions have been proposed in the context of routing and other functions in ad hoc networks. However, most of these address only unicast and multicast.

- The mechanism design solutions proposed in the literature are all based on the VCG mechanisms. Though the VCG mechanisms guarantee the extremely desirable property of dominant strategy incentive compatibility, the incentive budgets required tend to be very high. In fact, there is a recent study [33] on the limitations of VCG mechanisms (i.e. dominant strategy incentive compatible mechanisms) in the design of protocols for networks with selfish nodes.

- There are not too may studies in the literature which address the incentive compatible broadcast problem. Also the existing approaches for incentive compatible broadcast have many limitations. Further, the existing solutions available for unicast and multicast invariably lead to inefficient solutions when applied to the broadcast setting.

Till this point, we have set the stage for the need of designing efficient incentive based mechanisms for ICB problem. We propose to use Bayesian model to design such incentive mechanisms. In the following section, we argue in the favor of choosing a Bayesian model with which we can overcome several limitations due to VCG based mechanisms.

\section{Need for a Bayesian Model}

There are several reasons why the Bayesian model makes more sense than a VCG type of model:

- The payments in the VCG model are almost always much higher when compared with the Bayesian models (This is natural because the dominant strategy incentive compatibility property guaranteed by the VCG model is a much stronger property and hence entails higher incentives to be paid). This is well known in the mechanism design literature.

- In the case of the Bayesian model, budget balance is satisfied. On the other hand, in the case of the VCG model, budget balance is difficult to achieve, except under very special settings. Budget balance is a desirable property because it ensures that the protocol does not require any external source of funding and therefore is self-sustaining.

- In the case of broadcast, the context is such that there is one source node and the rest of the nodes are receivers of the broadcast packet. Since the nodes are rational, they may need to make payments to receive the broadcast packet(s). It makes sense to expect all the nodes that receive packets to make the same payments. This is because the source node has no way of distinguishing among the remaining nodes (which are all receivers). We have shown in this paper that the payments as computed by the Bayesian model will be identical for all the receiving nodes. Thus the Bayesian model captures the real-world in a natural way.

However, there are two issues with the use of Bayesian approach. First, due to the high payments entailed by VCG-based solutions, there is a compelling need to look for Bayesian 
incentive compatibility, which is a much weaker form of incentive compatibility. Second, we may lose out on individual rationality. We have however derived a sufficient condition under which individual rationality is also guaranteed by our Bayesian model.

\section{Contributions and Outline of the Paper}

Motivated by the above considerations, we offer the following contributions in this paper.

- We propose an incentive based broadcast mechanism that satisfies Bayesian incentive compatibility and minimizes the incentive budgets required by the individual nodes. Bayesian incentive compatibility ensures that truth revelation is a best response for each node whenever all other nodes are also truthful.

- The above proposed mechanism, which we call $B I C-B$ (Bayesian incentive compatible broadcast) mechanism, also satisfies budget balance. This ensures that the protocol is self-sustaining and does not require any external budget for sustaining the running of the protocol.

- We also derive a necessary and sufficient condition for the ex-post individual rationality of the BIC-B mechanism. Individual rationality guarantees that the nodes do not incur negative payoffs by participating in the protocol.

- We present an approach for a protocol implementation based on the BIC-B mechanism. We also explain how the payments to the nodes are computed following the proposed mechanism.

- We show that the $B I C-B$ protocol exhibits superior performance in comparison to existing protocols for incentive compatible broadcast such as DSIC-B.

The rest of the paper is organized as follows. In Section II, we present a game theoretic model for the incentive compatible broadcast problem and offer a Bayesian incentive compatible mechanism design solution for the problem. In Section III, we investigate different properties of the proposed BIC-B protocol such as budget balance, budget minimization, and individual rationality. In Section IV, we present a simulation experiment to exhibit the superior performance of the BIC-B protocol. Section V concludes the paper and outlines a few directions for future work.

\section{The Model AND the BIC-B PROtocol}

An ad hoc wireless network, in this paper, is represented by an undirected graph $G=(N, E)$, where $N=\{1,2, \ldots, n\}$ is the set of $n$ wireless nodes and $E$ is the set of communication links between the nodes. There exists a communication link between two nodes $i$ and $j$, if a node $i$ is reachable from node $j$, and node $j$ is also reachable from node $i$. Thus we have an undirected graph representation. We assume that wireless nodes use directional antennas and a single transmission by a node may be received by only a subset of nodes in its vicinity. We note that all nodes in the graph $G$ are connected.

We assume that the nodes in the ad hoc wireless network are owned by rational and intelligent individuals and so their objective is to maximize their individual goals. For this reason, they may not always participate loyally in key network functions, such as forwarding the packets, since such activity might consume the node's resources, such as battery power, bandwidth, CPU cycles, etc. Let each node $i$ incur a cost $\theta_{i}$ for forwarding a packet. For simplicity, we assume that $\theta_{i}$ is independent of the neighbor from which the packet is received and the neighbor to which the packet is destined. We can represent $\theta_{i}$ as the weight of node $i$ in the graph $G$. This implies that we work with a node weighted graph.

Consider the task of broadcast in such a setting. Assume that the source of the broadcast is node $s$. Not all remaining nodes may be connected to node $s$, hence appropriate intermediate nodes have to forward the broadcast packet to ensure that the packet reaches all the nodes in the network. As explained above, the nodes that forward the packet(s) incur costs. This means, we need to look at a tree that spans all the nodes and has the source node $s$ as the root of the tree. We call such a tree as Source Rooted Broadcast Tree (SRBT). Given an SRBT, we design an appropriate incentive scheme or mechanism that is built into the broadcast protocol.

\section{A. A Game Theoretic Model}

A game theoretic model in the above setting is described below.

- There are $n$ wireless nodes, $1,2, \ldots, n$ in the ad hoc network. Let $N=\{1,2, \ldots, n\}$.

- Each wireless node $i$ privately observes a signal $\theta_{i}$ that determines the cost for the node $i$ to forward a packet. The value of $\theta_{i}$ is known to agent $i$ deterministically since $\theta_{i}$ is dependent of the consumed CPU cycles, battery power, bandwidth, etc. However this value is not known to other nodes. Hence we call $\theta_{i}$ as the private value or type of node $i$.

- We denote by $\Theta_{i}$ the set of types of node $i, i=$ $1,2, \ldots, n . \Theta=\times_{i \in N} \Theta_{i}$ is the set of all type profiles of the nodes. $\theta=\left(\theta_{1}, \theta_{2}, \ldots, \theta_{n}\right)$ represents a typical type profile of the nodes.

- It is assumed that the types of the nodes are drawn from a common prior distribution $\varphi$. We make the standard assumption that individual belief functions $p_{i}$ are computed using the above common prior. The belief function $p_{i}(i=1,2, \ldots, n)$ describes the belief of node $i$ about the types of the remaining nodes.

- $X$ is the set of different possible outcomes. Each outcome specifies the set of routers and payments to those routers for forwarding packets. The selection of a particular choice of set of routers depends on the type profile $\theta=\left(\theta_{1}, \theta_{2}, \ldots, \theta_{n}\right)$ of the nodes. This is captured by social choice function $f($.$) [22].$

- If a node $i$ forwards a packet, it incurs a $\operatorname{cost} \theta_{i}$ and hence it must be compensated appropriately. More generally, we capture this notion by a utility function $u_{i}: X \times \Theta_{i} \rightarrow$ $R$. In particular, we assume that the utility functions are quasi-linear.

Following the terminology of mechanism design [22], a mechanism $M=\left(S_{1}, S_{2}, \ldots, S_{n}, g().\right)$ is a collection of strategy sets $S_{1}, S_{2}, \ldots, S_{n}$ and an outcome function $g($.$) . Here S_{i}$ captures the possible announcements of node $i$ regarding its incurred costs. The outcome function $g($.$) is defined as$ $g: S_{1} \times S_{2} \times \ldots \times S_{n} \rightarrow X$. A mechanism is a framework 
which prescribes an action set for each player and specifies how these action profiles are transformed into outcomes. The outcome function $g($.$) gives the rule for obtaining out-$ comes from action profiles. A mechanism induces a Bayesian game $\left(N,\left(S_{i}\right),\left(\Theta_{i}\right),\left(p_{i}\right),\left(U_{i}\right)\right)$ where $U_{i}($.$) is computed from$ $u_{i}\left(g(),. \theta_{i}\right)$ [22]. This induced Bayesian game can have a solution which is either a dominant strategy equilibrium or a Bayesian Nash equilibrium. Accordingly we have either a dominant strategy incentive compatible mechanism or a Bayesian incentive compatible mechanism, respectively.

\section{B. The BIC-B Protocol}

We now present a mechanism that implements the social choice function (SCF) $f(\theta)=\left(k(\theta), t_{1}(\theta), \ldots, t_{n}(\theta)\right)$, $\forall \theta \in \Theta$. Here $k(\theta)$, and $t_{i}(\theta), \forall i \in N$ are interpreted in the following way. $k(\theta)$ is the allocation rule that represents which nodes in the network have to forward the packet, given the profile $\theta$ of types. The vector $\left(t_{1}(\theta), \ldots, t_{n}(\theta)\right)$ gives payments received by the nodes, given the profile $\theta$ of types. For any $i \in N$, if $t_{i}(\theta)>0$, then the interpretation is that $i$ receives some positive amount and if $t_{i}(\theta)<0$, then the interpretation is that $i$ pays some positive amount.

Assume that we are given the $S R B T$ corresponding to the graph under consideration. We design, based on the given $S R B T$, an the following payment scheme that determines the payments $\left(t_{i}(\theta)\right)_{i \in N}$ to the individual nodes for a broadcast. In the SRBT, all the internal nodes forward the broadcast packet. We call such packet forwarding nodes as routers, and represent the set of routers by $R$. Note that each outcome of the SCF $f($.$) has an allocation rule and a payment rule. We define the$ allocation rule in the following way: $\forall \theta \in \Theta, \forall i \in N$,

$$
\begin{aligned}
k_{i}(\theta) & =1, \quad \text { if } i \in R \\
& =0, \quad \text { if } i \notin R
\end{aligned}
$$

The valuation function, $v_{i}\left(k(\theta), \theta_{i}\right)$, of node $i$ is its cost to forward a transit packet. From the allocation rule $k($.$) in our$ SCF $f($.$) , we get, \forall \theta \in \Theta, \forall i \in N$,

$$
\begin{aligned}
v_{i}\left(k(\theta), \theta_{i}\right) & =-\theta_{i}, \quad \text { if } i \in R \\
& =0, \quad \text { if } i \notin R
\end{aligned}
$$

The broadcast packets from the source node travel through the paths specified in the SRBT. To compensate the incurred cost of the routers in the network, we need to determine payments to the nodes. We follow the payment rule of the classical dAGVA mechanism or expected externality mechanism [22], [34], to compute the payments to the nodes in our scheme. Using the payment rule of the dAGVA mechanism [22], [34], $\forall i \in N, \forall \theta \in \Theta$, we get

$$
\begin{aligned}
t_{i}(\theta)= & E_{\theta_{-i}}\left[\sum_{l \neq i} v_{l}\left(k(\theta), \theta_{l}\right)\right] \\
& -\left(\frac{1}{n-1}\right) \sum_{j \neq i} E_{\theta_{-j}}\left[\sum_{l \neq j} v_{l}\left(k(\theta), \theta_{l}\right)\right]
\end{aligned}
$$

From (1), (2) we get, $\forall i \in N, \forall \theta \in \Theta$,

$t_{i}(\theta)=\left(\frac{1}{n-1}\right) \sum_{j \neq i} E_{\theta_{-j}}\left[\sum_{l \in R, l \neq j} \theta_{l}\right]-E_{\theta_{-i}}\left[\sum_{l \in R, l \neq i} \theta_{l}\right]$

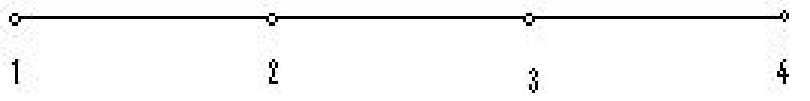

Fig. 2. Illustrative example 1

where $E_{\theta_{-i}}\left[\sum_{l \in R, l \neq i} \theta_{l}\right]$ is interpreted as the total expected value to node $i$ that would be generated by all the remaining nodes in the absence of node $i$. This completes the characterization of the payment rule of the BIC-B mechanism. Note that this mechanism with the above payment rule is incentive compatible. This directly follows from the incentive compatibility property of the dAGVA mechanism [22], [34].

In the following, we first present an illustrative example to understand the details of the proposed payment scheme and we then investigate the properties of the mechanism.

\section{An Example}

Now we provide an example to illustrate the payment scheme in $B I C-B$ protocol. Let us consider the graph in Figure 2, which is not bi-connected. We recall that the types of nodes are their incurred transit costs. We consider the type sets of nodes as $\Theta_{1}=\{10,11\}, \Theta_{2}=\{15,16\}$, $\Theta_{3}=\{12,13\}, \Theta_{4}=\{7,8\}$. Now let us assume that the players belief probability functions are independent discrete uniform distributions with equal probabilities for all types. and $\theta=(10,15,13,8)$ is the announced cost profile.

In this example, $S R B T$ is also the same the original graph. Now, the allocation rule is $k(\theta)=(0,1,1,0)$. We note that $N=\{1,2,3,4\}$ and $R=\{2,3\}$. The valuation functions of nodes are, $v_{1}(k(\theta))=0, v_{2}(k(\theta))=-15, v_{3}(k(\theta))=$ $-13, v_{4}(k(\theta))=0$. Now we compute the payments using the payment rule (3) of BIC-B protocol.

The payment computation for node 1 :

$$
\begin{aligned}
t_{1}(\theta)= & \left(\frac{1}{4-1}\right) \sum_{j \neq 1} E_{\theta_{-j}}\left[\sum_{l \in R, l \neq j} \theta_{l}\right] \\
& -E_{\theta_{-1}}\left[\sum_{l \in R, l \neq 1} \theta_{l}\right] \\
= & \left(\frac{1}{3}\right) E_{\theta_{-2}}\left[\sum_{l \in R, l \neq 2} \theta_{l}\right] \\
& +\left(\frac{1}{3}\right) E_{\theta_{-3}}\left[\sum_{l \in R, l \neq 3} \theta_{l}\right] \\
& +\left(\frac{1}{3}\right) E_{\theta_{-4}}\left[\sum_{l \in R, l \neq 4} \theta_{l}\right] \\
& -E_{\theta_{-1}}\left[\sum_{l \in R, l \neq 1} \theta_{l}\right] \\
= & \left(\frac{1}{3}\right)\left[E_{\theta_{-2}}\left[\theta_{3}\right]+E_{\theta_{-3}}\left[\theta_{2}\right]+E_{\theta_{-4}}\left[\theta_{2}+\theta_{3}\right]\right] \\
& -E_{\theta_{-1}}\left[\theta_{2}+\theta_{3}\right] \\
= & \left(\frac{1}{3}\right)\left[E_{\theta_{-2}}\left[\theta_{3}\right]+E_{\theta_{-3}}\left[\theta_{2}\right]\right] \\
& +\left(\frac{1}{3}\right)\left[E_{\theta_{-4}}\left[\theta_{2}\right]+E_{\theta_{-}}\left[\theta_{3}\right]\right] \\
& -\left[E_{\theta_{-1}}\left[\theta_{2}\right]+E_{\theta_{-1}}\left[\theta_{3}\right]\right] \\
& (\text { since types are statistically independent) } \\
= & \left(\frac{1}{3}\right)[12.5+15.5+15.5+12.5] \\
= & -[15.5+12.5] \\
& -9.33
\end{aligned}
$$

In the similar fashion, we can compute the payments to the remaining nodes also. The payments to nodes are: $t_{1}(\theta)=-9.3, t_{2}(\theta)=11.3, t_{3}(\theta)=7.3, t_{4}(\theta)=-9.3$. In this example, node 1 and node 4 do not forward the packets. Observe that they pay the same amount, namely 9.33 . Since node 2 and node 3 are routers, they receive amounts 11.33 and 7.33 respectively. Further observe that $\sum_{i=1}^{4} t_{i}(\theta)=0$. 


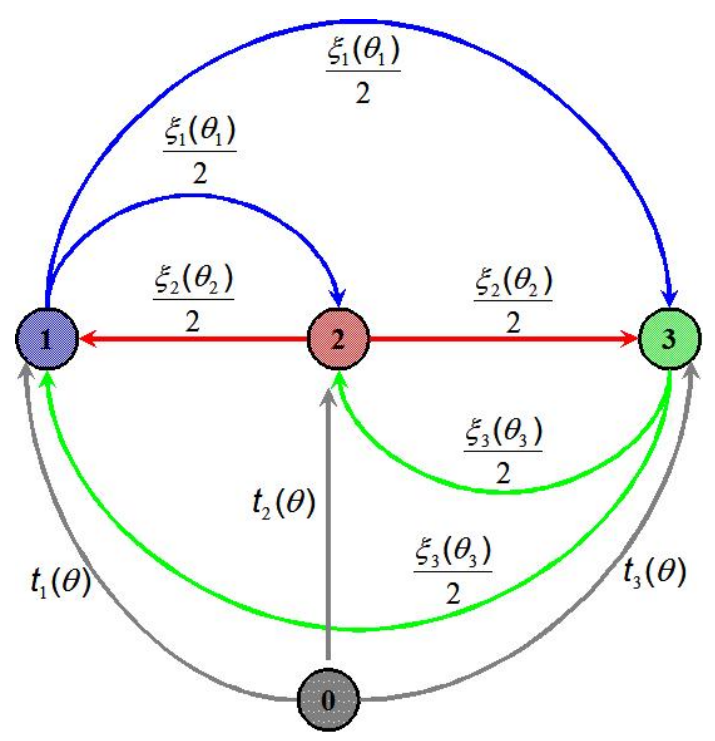

Fig. 3. An interpretation of the payment rule

\section{Properties of the BIC-B Protocol}

\section{A. Budget Balance}

Assume that $\xi_{i}\left(\theta_{i}\right)=E_{\theta_{-i}}\left[\sum_{l \in R, l \neq i} \theta_{l}\right], \forall i \in N$. To show budget balance, we need to show that the sum of the payments received and the payments made by the nodes in the network is zero, i.e. $\sum_{i} t_{i}(\theta)=0, \forall \theta \in \Theta$. Let us assume that $\xi_{i}\left(\theta_{i}\right)=E_{\theta_{-i}}\left[\sum_{l \in R, l \neq i} \theta_{l}\right], \forall \theta \in \Theta, \forall i \in N$. Then, from (3), we get $\forall \theta \in \Theta$

$$
\begin{aligned}
t_{i}(\theta) & =\xi_{i}\left(\theta_{i}\right)-\left(\frac{1}{n-1}\right) \sum_{j \neq i} \xi_{j}\left(\theta_{j}\right) \\
\text { then, } & \\
\sum_{i=1}^{n} t_{i}(\theta)= & \sum_{i=1}^{n} \xi_{i}\left(\theta_{i}\right)-\left(\frac{1}{n-1}\right) \sum_{i=1}^{n} \sum_{j \neq i} \xi_{j}\left(\theta_{j}\right) \\
= & \sum_{i=1}^{n} \xi_{i}\left(\theta_{i}\right)-\left(\frac{1}{n-1}\right) \sum_{i=1}^{n}(n-1) \xi_{j}\left(\theta_{j}\right) \\
& \Rightarrow \quad \sum_{i=1}^{n} t_{i}(\theta)=0
\end{aligned}
$$

It can be noted that each node $i$ distributes $\xi_{i}$ equally among the remaining $(n-1)$ nodes. Figure 3 shows this interpretation for a graph with 3 nodes.

Let us represent the flow into the node with positive sign and the flow going out from the node by negative sign. Now the payment, $t_{1}(\theta)$, for node 1 can be written as

$$
t_{1}\left(\theta_{1}, \theta_{2}, \theta_{3}\right)=\frac{1}{2} \xi_{2}\left(\theta_{2}\right)+\frac{1}{2} \xi_{3}\left(\theta_{3}\right)-\xi_{1}\left(\theta_{1}\right)
$$

Similarly, we can write down the payments to the remaining two nodes.

\section{B. Payments by Non-Router Nodes}

Recall that $N$ is the set of nodes and $R$ represents the set of routers in the network. We now state Lemma 1 which is useful in proving Lemma 2 and subsequently Theorem 1.

Lemma 1: For any $i \in R$ and for any $j \notin R$, we have

$$
E_{\theta_{-i}}\left[\sum_{l \in R, l \neq i} \theta_{l}\right]=E_{\theta_{-j}}\left[\sum_{l \in R, l \neq i} \theta_{l}\right] .
$$

Proof: Proof is provided in the appendix.

Lemma 2: For the BIC-B protocol, $t_{i}(\theta)<0, \forall i \notin R, \forall \theta \in$ $\Theta$. That is, the nodes other than the routers will pay a positive amount of money for receiving the packet(s).

Proof: If $R$ is empty, then the source node can reach all the remaining nodes in the network within a single hop. In that case, the payments will be 0 . We are not interested in such a trivial situation. So we assume that,

$$
|R|>0
$$

Let us assume that, $\forall j \notin R$,

$$
\begin{aligned}
& \Gamma_{j}=E_{\theta_{-j}}\left[\sum_{l \in R, l \neq j} \theta_{l}\right] \\
& =E_{\theta_{-j}}\left[\sum_{l \in R} \theta_{l}\right] \quad \text { (since } j \notin R \text { ) }
\end{aligned}
$$

Since the types of nodes are statistically independent, the values of $\Gamma_{j}, \forall j \notin R$, are all the same. We represent this value with $\Gamma$. That is,

$$
\Gamma=\Gamma_{j}, \quad \forall j \notin R .
$$

Now, let us assume that $\Upsilon_{i}=E_{\theta_{-i}}\left[\sum_{l \in R, l \neq i} \theta_{l}\right], \forall i \in R$. Then

$$
\begin{aligned}
\Upsilon_{i}= & E_{\theta_{-i}}\left[\sum_{l \in R, l \neq i} \theta_{l}\right] \\
= & E_{\theta_{-j}}\left[\sum_{l \in R, l \neq i} \theta_{l}\right] \\
& \text { (consequence of Lemma 1) } \\
< & \left.E_{\theta_{-j}}\left[\sum_{l \in R} \theta_{l}\right] \quad \text { (since } i \in R\right) \\
= & \Gamma \quad \text { (from equation (5)) }
\end{aligned}
$$

So, we can conclude that

$$
\Upsilon_{i}<\Gamma, \quad \forall i \in R
$$

From the payment rule (3) of the $B I C-B$ protocol, we have $\forall i \notin R$,

$$
\begin{aligned}
t_{i}(\theta)= & \left(\frac{1}{n-1}\right) \sum_{j \neq i, j \in N} E_{\theta_{-j}}\left[\sum_{l \in R, l \neq j} \theta_{l}\right] \\
& -E_{\theta_{-i}}\left[\sum_{l \in R, l \neq i} \theta_{l}\right] \\
= & \left(\frac{1}{n-1}\right) \sum_{j \in R} E_{\theta_{-j}}\left[\sum_{l \in R, l \neq j} \theta_{l}\right] \\
& +\left(\frac{1}{n-1}\right) \sum_{j \neq i, j \notin R} E_{\theta_{-j}}\left[\sum_{l \in R, l \neq j} \theta_{l}\right] \\
& -E_{\theta-i}\left[\sum_{l \in R, l \neq i} \theta_{l}\right] \\
= & \left(\frac{1}{n-1}\right) \sum_{j \in R} \Upsilon_{j}+\left(\left(\frac{1}{n-1}\right) \sum_{j \neq i, j \notin R} \Gamma\right)-\Gamma \\
& (\text { since from equation }(5)) \\
= & \left(\frac{1}{n-1}\right) \sum_{j \in R} \Upsilon_{j}+\left(\frac{|N|-|R|-1}{n-1}-1\right) \Gamma \\
= & \left(\frac{1}{n-1}\right) \sum_{j \in R} \Upsilon_{j}-\left(\frac{1}{n-1}\right) \sum_{j \in R} \Gamma \\
= & \left(\frac{1}{n-1}\right) \sum_{j \in R}\left(\Upsilon_{j}-\Gamma\right) \\
< & 0, \quad \text { (from }(6)) .
\end{aligned}
$$

According to our previous interpretation, $t_{i}(\theta)<0$ means that node $i$ needs to pay the specified amount. This completes the proof the lemma. (Q.E.D.).

Observation 1: From the proof of Lemma 2, we know that $\forall i \notin R$, 


$$
t_{i}(\theta)=\left(\frac{1}{n-1}\right) \sum_{j \in R}\left(\Upsilon_{j}-\Gamma\right)
$$

Note that the right hand side of the above expression is independent from $i$. Hence, using the BIC-B protocol, $t_{i}($.$) ,$ $\forall i \notin R$ are all the same. The immediate implication is that the payments made by the nodes other than the routers are the same.

\section{Optimality of the BIC-B Payments}

Here we prove the optimality of the payments prescribed by the $B I C-B$ protocol. We note that an appropriate allocation rule is employed to determine the SRBT of the underlying graph of the ad hoc wireless network for the broadcast task. We define cost of SRBT is sum of the forwarding costs of the router nodes. It is the case that any allocation rule tries to minimize the cost of the SRBT. If it is hard to find optimal SRBT, then we assume that an appropriate approximation algorithm is used to determine the SRBT.

We make the following observation for the sake of Theorem 1.

Observation 2: Consider two type profiles $\theta$ and $\theta^{\prime}$. Assume that these two types are different only with respect to the type of node $i$. Using the BIC-B mechanism, when the types are $\theta$ and $\theta^{\prime}$, the payments to node $i$ are $t_{i}(\theta)$ and $t_{i}\left(\theta^{\prime}\right)$ respectively. Note that if the corresponding SRBT is the same for both the types $\theta$ and $\theta^{\prime}$, then the set of routers is the same and hence the payments $t_{i}(\theta)$ and $t_{i}\left(\theta^{\prime}\right)$ respectively to node $i$ are the same. This is because in the payment rule (3) of BIC-B mechanism, the quantities involved only look for expected values of the types of the routers.

Theorem 1: For the given SRBT structure of the underlying graph $G$ of the ad hoc wireless network, the payment to any node using the BIC-B mechanism is minimum among all other Bayesian incentive compatible mechanisms based on SRBT.

Proof: We provide a contradiction to prove the statement. Let $t($.$) be the payment rule of the B I C-B$ mechanism based on the given SRBT. Assume that there exists another Bayesian incentive compatible mechanism with payment rule $\hat{t}(\cdot)$ such that the payment to a router node $i$ is strictly less. That is,

$$
\hat{t}_{i}(\theta)<t_{i}(\theta), \quad \forall \theta \in \Theta, \text { for some } i \in N
$$

We construct a contradiction to show that $\hat{t}(\cdot)$ is not incentive compatible for the node $i$ under some cost profile $\theta^{\prime}$. We construct $\theta^{\prime}$ from $\theta$ by replacing the $\operatorname{cost} \theta_{i}$ of node $i$ with $\left(t_{i}(\theta)+\epsilon\right)$, where $\epsilon>0$. That is, if the cost profile $\theta$ is such that $\theta=\left(\theta_{i}, \theta_{-i}\right)$, (where the $n$-tuple $\left(\theta_{i}, \theta_{-i}\right)$ indicates a cost profile of the nodes where the cost of node $i$ is $\theta_{i}$ and the costs of remaining nodes is represented by $\theta_{-i}$ ), then the cost profile $\theta^{\prime}$ is such that $\theta^{\prime}=\left(\left(t_{i}(\theta)+\epsilon\right), \theta_{-i}\right)$. Recall that node $i$ is a router in the corresponding SRBT when type profile is $\theta$. We now consider the following two cases.

Case 1: Consider $\theta^{\prime}$ is the announced cost profile. Assume that node $i$ is a router in the corresponding SRBT. This assumption is true definitely if $\left(t_{i}(\theta)+\epsilon\right)<\theta_{i}$ because node $i$ is a router even with the type $\theta_{i}$. That is, the SRBT is one and the same under both the type profiles $\theta$ and $\theta^{\prime}$. Using the payment rule $\hat{t}($.$) , the payment to node i$ is $\hat{t}_{i}\left(\theta^{\prime}\right)$. Now the gain from being a router to the node $i$ is $\hat{t}_{i}\left(\theta^{\prime}\right)-\left(t_{i}(\theta)+\epsilon\right)<t_{i}\left(\theta^{\prime}\right)-\left(t_{i}(\theta)+\epsilon\right)=-\epsilon<0$ (using (7) and Observation 2).

Case 2: Consider $\theta^{\prime}$ is the announced cost profile. Assume that node $i$ is not a router in the appropriate SRBT. Then there is no forwarding cost incurred to node $i$. Then there is no issue.

Hence for node $i$, if its type is $\left(t_{i}(\theta)+\epsilon\right)<\theta_{i}$ and then it happens to be a router in the corresponding SRBT and it gets negative gains. Due to the appropriate arguments in Theorem 2 , there exists a $\theta_{i} \in \Theta_{i}$ such that $\left(t_{i}(\theta)+\epsilon\right)<\theta_{i}$ is true. Hence the mechanism with $\hat{t}(\cdot)$ as the payment rule is not incentive compatible. This provides the required contradiction. (Q.E.D.).

\section{Individual Rationality of the BIC-B Protocol}

We now investigate the individual rationality $(I R)$ of the $B I C$ - $B$ protocol. In particular, we investigate the ex post individual rationality, which is the strongest among the three notions of individual rationality [22], [32]. In the following theorem, we obtain a necessary and sufficient condition for the ex post individual rationality of the BIC-B protocol. Let $\hat{\theta}_{i}$ be the announced cost of a node $i$ and $\theta_{i}$ be the actual cost of that node.

Theorem 2: The BIC-B protocol is ex post individual rational if and only if

$$
\hat{\theta}_{i} \leq\left(\frac{n}{n-1}\right) E\left[\theta_{i}\right], \quad \forall i \in R
$$

where the $\hat{\theta}_{i}$ is the announced cost of the node $i$.

Proof: See Appendix.

\section{E. An Implementation of the BIC-B Protocol}

In the previous sections, we have designed the BIC-B mechanism for providing incentives to the nodes to participate in the broadcast task. We have also proved certain important properties of the mechanism. Here we provide a protocol implementation for the BIC-B mechanism.

The protocol implementation is motivated by the emerging technology of hybrid ad hoc wireless networks, where there are base stations that provide fixed infrastructure for many network related functions. We propose that additional functionality (which we call mediation functionality) as described below be incorporated into each base station. Let us call this part of the base station as the mediator. The mediator could be a part of any other network infrastructure also.

The mediator elicits the types from all the nodes, computes the allocation and payments of the nodes, and announces the outcome. We assume that all the nodes can communicate with the mediator.

After receiving the messages from the mediator regarding the payments, each node constructs an internal table as shown in Table I. Each row of the table corresponds to a node in the network. Each row contains three fields of information: (a) Source ID, which specifies the source node ID from which the packet is originating, (b) Node List, which specifies the set of nodes to which the packet needs to be forwarded, and (c) 
TABLE I

STRUCTURE OF INTERNAL TABLE OF A NODE

\begin{tabular}{|l|l|l|}
\hline Source ID & Node List & Payment \\
\hline & & \\
\hline & & \\
\hline
\end{tabular}

Payment, which specifies the payment to be received or paid. The following is the structure of internal table of a node:

In view of the above, the BIC-B protocol can be implemented as follows.

BIC-B Protocol: If a node receives a broadcast packet, it checks its internal table entry corresponding to the source ID of the broadcast. Then it forwards the packet to the set of nodes specified in the Node List field of the entry and receives the payment as mentioned in the Payment field of the entry. On the other hand, if the Node List field is empty, then the node does not forward the packet to any node and makes a payment as mentioned in the Payment field of the entry.

In the above protocol, all the payment information by the node is communicated directly to the mediator which takes care of all the book keeping.

\section{Performance of the BIC-B Protocol}

In this section, we show the efficacy of the proposed $B I C-B$ protocol for the ICB problem. In our simulation experiments, we compare the performance of the $B I C-B$ protocol with that of the Dominant Strategy Incentive Compatible Broadcast (DSIC-B) protocol [11], [30].

\section{A. Simulation Model}

The DSIC-B protocol is based on dominant strategy equilibrium of the underlying game and the $B I C-B$ protocol is based on the Bayesian Nash equilibrium of the underlying game. Since every dominant strategy equilibrium is also a Bayesian Nash equilibrium, but not vice-versa, we first find a dominant strategy equilibrium of the underlying game and compute the payments to the nodes using the DSIC-B and the $B I C-B$ protocols [11].

We work with a randomly generated graph of an ad hoc wireless network with the number of nodes $n=$ $5,10,15,20,25,30,35,40$. According to our network model presented in Section II, the graph is node weighted where these weights are the transit costs of the nodes chosen independently and uniformly from a range $[1,50]$. After the nodes announce their types, we first compute the least cost paths to all the nodes from the source node and then construct an SRBT. Using the SRBT, we can decide the set of routers. This fixes the allocation rule. Then we compute payments to the nodes using the payment rule of the appropriate broadcast protocol. In all our simulation experiments, the results for the performance metrics are averages taken over 100 random instances.

\section{B. Simulation Results}

We consider two performance metrics. The first metric is average payment to routers (APR). This specifies the payment on an average to each router for forwarding the transit packets.

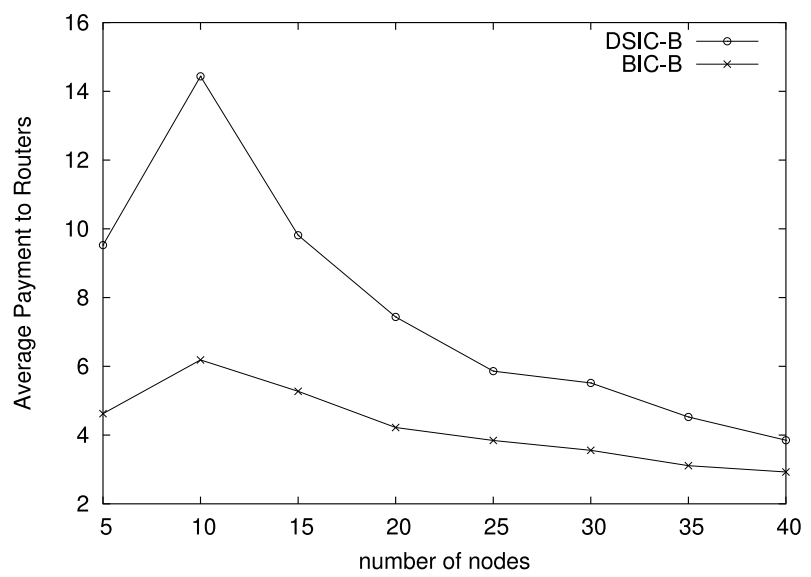

Fig. 4. Average payment to routers in the DSIC-B and $B I C-B$ protocols

The graph in Figure 4 shows the comparison of the BIC-B protocol and the DSIC-B protocol using APR. In the figure, the lower curve corresponds to the $B I C-B$ protocol. It is clear from the figure that the $B I C-B$ protocol performs better than the $D S I C-B$ protocol. This means that the system wide payments made by the nodes to forward a broadcast packet is less using the $B I C-B$ protocol.

The second performance metric, we follow, is the worst overpayment ratio (WOR). We first define overpayment ratio as the ratio of payment made by a node to its least cost path value from the source node $s$. Now, we can define WOR as the maximum over the overpayment ratios of all the nodes in the network. That is,

WOR $=\max _{i \in N} \frac{\text { payment made by node } \mathrm{i}}{\text { cost of path from source of broadcast to node } i}$

where cost of path from source of broadcast to node $i$ is the sum of forwarding costs of the nodes that lie on the path from the source of broadcast node to the node $i$. Ideally we expect this ratio to be 1 . We compare the WOR of $B I C-B$ protocol and the DSIC-B protocol in Figure 5. We note that the lower curve corresponds to the $B I C-B$ protocol in the figure. From the graph, it is easy to see that the worst overpayment ratio in the network is higher using the DSIC-B protocol than BIC-B protocol. WOR conveys the following significant information. When a node receives a packet from a router, then clearly the payment made by the receiver node to the router is higher than the value of its least cost path, since it has to give incentives to the router to make it reveal the true incurred cost. If we take a ratio of the payment to the value of least cost path, from the Figure 5, this ratio is less than 2 times over all the nodes using the $B I C-B$ protocol and it is higher than 5 times over all the nodes using the $D S I C-B$ protocol. This says that nodes end up with very high payments, using $D S I C-B$ protocol, than actually what their value of the corresponding least cost path.

\section{CONCLUSions AND Future Work}

We considered the incentive compatible broadcast (ICB) problem in ad hoc wireless networks with selfish nodes. We proposed an incentive based broadcast protocol that satisfies Bayesian incentive compatibility and minimizes the incentive 


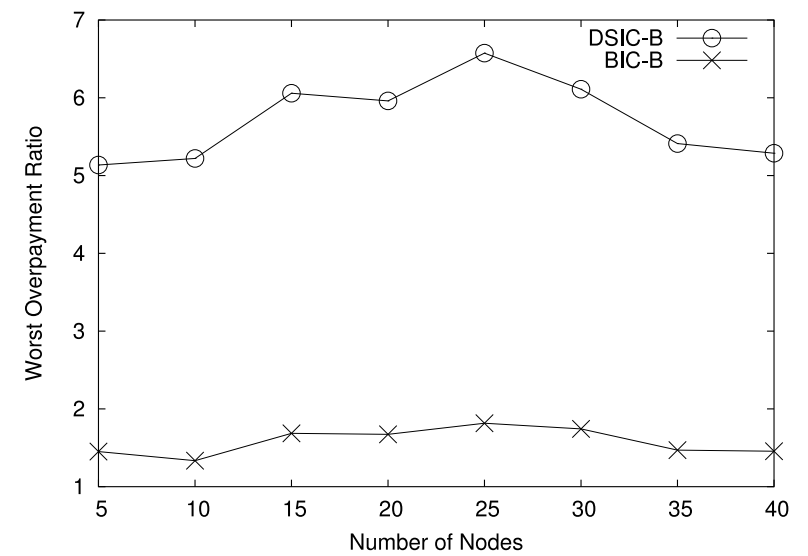

Fig. 5. Worst overpayment ratios for the DSIC-B and BIC-B protocols

budgets required by the individual nodes. The proposed protocol, $B I C-B$, also satisfies budget balance. We also derived a necessary and sufficient condition for the ex-post individual rationality of the BIC-B protocol. We showed that the BIC$B$ protocol exhibits superior performance when compared to a dominant strategy incentive compatible solution to the problem. Thus in this paper, we have addressed the ICB problem by proposing an incentive mechanism and design of the BIC-B protocol.

While designing incentive based protocols, we note that the complete solution includes (a) design of the incentive mechanism (b) design of a protocol which implements the incentive mechanism and (c) addressing any problems that may arise, such as the cheating problem. Our main contribution in this paper is in designing an incentive mechanism. We have also briefly addressed the design of a protocol in the current version of the paper. To address the cheating, we need to invoke cryptographic techniques. There is some literature available on the use of cryptographic techniques to implement protocols, for example [35].

In terms of mechanism design, it would be interesting to explore optimal broadcast mechanisms in the Myerson sense [22], [34]. These are mechanisms that minimize the incentive budgets subject to Bayesian incentive compatibility and individual rationality.

An important problem that could be explored is design of Bayesian incentive compatible protocols for unicast and multicast problems. Existing game theoretic approaches to unicast and multicast are all based on VCG mechanisms and have the usual limitations associated with the use of VCG mechanisms.

Also, it is important to address certain practical issues that arise in the implementation of these mechanisms as part of standard protocols. For example, the payment computation is performed in a centralized way in the BIC-B protocol. It would be interesting to design a distributed algorithm for this problem that could help deploy the BIC-B protocol in the real world.

\section{ACKNOWLEDGMENT}

The research in this paper is partially supported by a research on Algorithmic Mechanism Design for Complex Game Theoretic Problems funded by the Office of Naval Research (Grant No. N0014-06-1-0994), Arlington, VA, USA. We wish to thank Dr Shantanu Das, Program Manager, Communications and Networking, ONR.

\section{APPENDIX}

\section{A. Proof of Lemma 1}

Note that the types of the nodes are statistically independent according to the current network model. For this reason, it does not matter even though we take the expectation, in the above expression, with respect to $\theta_{i}$ or $\theta_{j}$, where $i \in R$ and $j \notin R$. Now it is easy to prove the lemma. Let the nodes in the set $R$ be indexed by the set $\{1,2, \ldots, r\}$, where $r=|R|$. Now for any $j \notin R$ and $i \in R$,

$$
\begin{aligned}
& E_{\theta_{-j}}\left[\sum_{l \in R, l \neq i} \theta_{l}\right] \\
& =\int \ldots \int\left[\sum_{l \in R, l \neq i} \theta_{l}\right] q\left(x_{1}\right) \ldots q\left(x_{j-1}\right) q\left(x_{j+1}\right) \ldots q\left(x_{n}\right) \\
& d\left(x_{1}\right) \ldots d\left(x_{j-1}\right) d\left(x_{j+1}\right) \ldots d\left(x_{n}\right) \\
& =\int \ldots \int\left[\sum_{l \in R, l \neq i} \theta_{l}\right] q\left(x_{1}\right) \ldots q\left(x_{r}\right) \quad d\left(x_{1}\right) \ldots d\left(x_{r}\right) \\
& \text { (since types are statistically independent) } \\
& \begin{array}{c}
=\int \ldots \int\left[\sum_{l \in R, l \neq i} \theta_{l}\right] q\left(x_{1}\right) \ldots q\left(x_{i-1}\right) q\left(x_{i+1}\right) \ldots q\left(x_{r}\right) \\
d\left(x_{1}\right) \ldots d\left(x_{i-1}\right) d\left(x_{i+1}\right) \ldots d\left(x_{r}\right)
\end{array} \\
& \text { (since }\left[\sum_{l \in R, l \neq i} \theta_{l}\right] \text { does not include } \theta_{i} \text { ) } \\
& =\int \ldots \int\left[\sum_{l \in R, l \neq i} \theta_{l}\right] q\left(x_{1}\right) \ldots q\left(x_{i-1}\right) q\left(x_{i+1}\right) \ldots q\left(x_{n}\right) \\
& d\left(x_{1}\right) \ldots d\left(x_{i-1}\right) d\left(x_{i+1}\right) \ldots d\left(x_{n}\right) \\
& \text { (since types are statistically independent) } \\
& =E_{\theta_{-i}}\left[\sum_{l \in R, l \neq i} \theta_{l}\right] \\
& \text { Q.E.D. }
\end{aligned}
$$

\section{B. Proof of Theorem 2}

We show that the utility of each router is non-negative after participating in the mechanism if and only if the specified condition holds. This is nothing but proving the ex post individual rationality of the nodes.

Let $f$ be the mechanism for the ICB problem. Now for ex post individual rationality to hold for the router nodes,

$$
u_{i}\left(f(\hat{\theta}), \hat{\theta}_{i}\right) \geq 0, \forall \hat{\theta} \in \Theta, \quad \forall i \in R .
$$

where $\hat{\theta}$ is the vector of announcements of the costs (or types) of the nodes. We also call $\hat{\theta}$ as the announced cost (or type) profile of the nodes.

Now we characterize the utility of each node $i \in R$. All nodes are the intended recipients of the packet(s) in a broadcast in the network. We know, from Observation 1, that the payment made by a non-router node is equivalent to $t_{m}(\hat{\theta})$ for any $m \notin R$. Since the routers are also intended recipients, they also need to pay this amount. But, actually the routers do not pay this amount and hence it is credited to their utility. Now we have, $\forall \hat{\theta} \in \Theta$,

$$
u_{i}\left(f(\hat{\theta}), \hat{\theta}_{i}\right)=v_{i}\left(k\left(\hat{\theta}_{i}, \hat{\theta}_{-i}\right)\right)-t_{m}(\hat{\theta})+t_{i}(\hat{\theta})
$$


We have $t_{m}(\hat{\theta})<0$ for any $m \notin R$, from Lemma 2 . Hence this term appears with negative sign in the expression (8). Now substituting the expression (3) in (8) and rearranging the terms, we get $\forall \hat{\theta} \in \Theta$,

$$
\begin{aligned}
u_{i}\left(f(\hat{\theta}), \hat{\theta}_{i}\right) & =-\hat{\theta}_{i}+E_{\theta_{-m}}\left[\sum_{l \in R} \theta_{l}\right]-E_{\theta_{-i}}\left[\sum_{l \in R, l \neq i} \theta_{l}\right] \\
& +\left(\frac{1}{n-1}\right) \sum_{j \neq i} E_{\theta_{-j}}\left[\sum_{l \in R, l \neq j} \theta_{l}\right] \\
& -\left(\frac{1}{n-1}\right) \sum_{j \neq m} E_{\theta_{-j}}\left[\sum_{l \in R, l \neq j} \theta_{l}\right]
\end{aligned}
$$

By canceling out appropriate the terms, we get $\forall \hat{\theta} \in \Theta$,

$$
\begin{aligned}
u_{i}\left(f(\hat{\theta}), \hat{\theta}_{i}\right) & =-\hat{\theta}_{i}+E_{\theta_{-m}}\left[\sum_{l \in R} \theta_{l}\right]-E_{\theta_{-i}}\left[\sum_{l \in R, l \neq i} \theta_{l}\right] \\
& +\left(\frac{1}{n-1}\right) E_{\theta_{-m}}\left[\sum_{l \in R} \theta_{l}\right] \\
& -\left(\frac{1}{n-1}\right) E_{\theta_{-i}}\left[\sum_{l \in R, l \neq i} \theta_{l}\right] \\
& =-\hat{\theta}_{i}+E_{\theta_{-m}}\left[\sum_{l \in R} \theta_{l}\right]-E_{\theta-m}\left[\sum_{l \in R, l \neq i} \theta_{l}\right] \\
& +\left(\frac{1}{n-1}\right) E_{\theta_{-m}}\left[\sum_{l \in R} \theta_{l}\right] \\
& -\left(\frac{1}{n-1}\right) E_{\theta_{-m}}\left[\sum_{l \in R, l \neq i} \theta_{l}\right]
\end{aligned}
$$

(consequence of Lemma 1)

$$
\begin{aligned}
& =-\hat{\theta}_{i}+E_{\theta_{-m}}\left[\sum_{l \in R} \theta_{l}\right]-E_{\theta_{-m}}\left[\sum_{l \in R} \theta_{l}\right] \\
& +E_{\theta_{-m}}\left[\theta_{i}\right]+\left(\frac{1}{n-1}\right) E_{\theta_{-m}}\left[\sum_{l \in R} \theta_{l}\right] \\
& -\left(\frac{1}{n-1}\right) E_{\theta_{-m}}\left[\sum_{l \in R} \theta_{l}\right] \\
& +\left(\frac{1}{n-1}\right) E_{\theta_{-m}}\left[\theta_{i}\right]
\end{aligned}
$$

(by expanding the Expectation terms)

$$
\begin{aligned}
& =-\hat{\theta}_{i}+E\left[\theta_{i}\right]+\left(\frac{1}{n-1}\right) E\left[\theta_{i}\right] \\
& =\frac{n E\left[\theta_{i}\right]-(n-1) \hat{\theta}_{i}}{n-1}
\end{aligned}
$$

For the $B I C-B$ protocol to be ex post individually rational,

$$
u_{i}\left(f(\hat{\theta}), \hat{\theta}_{i}\right) \geq 0, \quad \forall \hat{\theta} \in \Theta, \quad \forall i \in R
$$

From the above characterization of utility function, we get

$$
\frac{n E\left[\theta_{i}\right]-(n-1) \hat{\theta}_{i}}{n-1} \geq 0, \quad \forall i \in R
$$

This implies,

$$
\hat{\theta}_{i} \leq\left(\frac{n}{n-1}\right) E\left[\theta_{i}\right], \quad \forall i \in R .
$$

Furthermore, it is easy to see that each of above arguments are reversible and hence the specified condition is necessary and sufficient for $B I C-B$ protocol to satisfy ex post individual rationality.

Q.E.D.

\section{REFERENCES}

[1] R. B. Myerson, Game Theory: Analysis of Conflict, Harvard University Press, Cambridge, Massachusetts, 1997.

[2] V. Srivastava, J. Neel, A.B. MacKenzie, R. Menon, L.A. DaSilva, J. Hicks, J.H. Reed, and R. Gilles, "Using game theory to analyze wireless ad hoc networks," IEEE Commun. Surveys and Tutorials, vol. 7, no. 4, pp. 46-56, 2005.

[3] I. Zaikiuddin, T. Hawkins, and N. Moffat, "Towards a game-theoretic understanding of ad-hoc routing," in Proc. Workshop on Games in Design and Verification (GDV), 2004, pp. 67-92.

[4] Q. Chen and Z. Niu, "A game-theoretical power and rate control for wireless ad hoc networks with step-up price," IEICE Trans. Commun., vol. E88-B, no. 9, pp. 3515-3523, 2005.

[5] C. Saraydar, N. Mandayam, and D. Goodman, "Nash equilibria of packet forwarding strategies in wireless ad hoc networks," IEEE Trans. Commun., vol. 50, no. 2, pp. 291-303, 2002.

[6] P. Kyasanur and N.H. Vaidya, "Selfish MAC layer misbehavior in wireless networks," IEEE Trans. Mobile Comput., vol. 4, no. 5, pp. 502-516, 2004

[7] Z. Fang and B. Bensaou, "Fair bandwidth sharing algorithms based on game theory frameworks for wireless ad-hoc networks," in Proc. 23rd IEEE Conference on Computer and Communications, (INFOCOM), 2004, pp. 1284-1295.

[8] B. Lu and U.W. Pooch, "A game theoretic framework for bandwidth reservation in mobile ad hoc networks," in Proc. 1st International Conference on Quality of Service in Heterogeneous Wired/Wireless Networks (QSHINE04), 2004.

[9] L. Anderegg and S. Eidenbenz, "Ad hoc-VCG: A truthful and costefficient routing protocol for mobile ad hoc networks with selfish agents," in Proc. 9th Annual ACM International Conference on Mobile Computing and Networking (MOBICOM), 2003, pp. 245-259.

[10] W. Wang and X.Y. Li, "Low-cost truthful multicast in selfish and rational wireless ad hoc networks," in Proc. 1st IEEE International Conference on Mobile Ad-hoc and Sensor Systems (MASS), 2004.

[11] N. Rama Suri, "Design of incentive compatible protocols for wireless ad hoc networks: A Game theoretic approch," in Proc. IEEE INFOCOM 2006 Student's Workshop, Barcelona, 2006.

[12] P. Resnick, R. Zeckhauser, E. Friedman, and K. Kuwabara, "Reputation systems: Facilitating trust in internet interactions," Commun. ACM, vol. 43 , no. 12 , pp. $45-48,2000$

[13] S. Marti, T.J. Giuli, K. Lai, and M. Baker, "Mitigating routing misbehaviour in mobile ad hoc networks," in Proc. 6th Аnnual ACM International Conference on Mobile Computing and Networking (MOBICOM), 2000, pp. 255-265.

[14] P. Michiardi and R. Molva, "Core: a collaborative reputation mechanism to enforce node cooperation in mobile ad hoc networks," in Proc. 6th Joint Working Conference on Communications and Multimedia Security: Advanced Communications and Multimedia Security, Kluwar, 2002, pp. $107-121$.

[15] S. Buchegger and J.-Y.L. Boudec, "Performance analysis of the confidant protocol: Cooperation of nodes - fairness in dynamic ad hoc networks," in Proc. 3rd ACM International Symposium on Mobile Ad Hoc Networking and Computing, 2002, pp. 226-236.

[16] V. Srinivasan, P. Nuggehalli, F. Chiasserini, and R.R. Rao, "Cooperation in wireless ad hoc networks," in Proc. 22nd IEEE Conference on Computer and Communications, (INFOCOM), 2003, pp. 808-817.

[17] E. Altman, A.A. Kherani, P. Michiardi, and R. Molva, "Non-cooperative forwarding in ad-hoc networks," Tech. Rep., INRIA, Sophin Antipolis, France, 2004.

[18] A. Urpi, M. Bonuccelli, and S. Giordano, "Modeling cooperation in mobile ad hoc networks: a formal description of selfishness," in Proc. 1st Workshop on Modeling and Optimiztion in Mobile, Ad Hoc and Wireless Networks, 2003.

[19] P. Nurmi, "Modelling routing in wireless ad hoc networks with dynamic bayesian games," in Proc. 1st IEEE Conference on Sensor, Mesh and Ad Hoc Communications and Networks (SECON), 2004, pp. 63-70. 
[20] J. Feigenbaum, C. Papadimitriou, R. Sami, and S. Shenker, "A BGP-based mechanism for lowest-cost routing," in Proc. 21st ACM Symposium on Principles Of Distributed Computing (PODC), 2002, pp. $173-182$.

[21] J. Hershberge and S. Suri, "Vickrey prices and shortest paths: What is an edge worth?," in Proc. 42th IEEE Symposium on Foundations of Computer Science (FOCS), 2001, pp. 252-259.

[22] A.S. Mas-Colell, M.D. Whinston, and J.R. Green, Microeconomic Theory, Oxford University Press, New York, 1995.

[23] D. Garg and Y. Narahari, "Foundations of mechanism design," Tech. Rep., Dept. of Computer Science and Automation, Indian Institute of Science, Bangalore, India, 2006.

[24] S. Eidenbenz, P. Santi, and G. Resta, "COMMIT: A sendercentric truthful and energy-efficient routing protocol for ad hoc networks," in Proc. International Workshop on Wireless, Mobile, and Ad Hoc Networks (WMAN) in conjunction with 19th IEEE International Parallel and Distributed Processing Symposium (IPDPS), 2005.

[25] W. Wang and X.Y. Li, "Truthful low-cost unicast in selfish wireless networks," in Proc. 4th International Workshop on Algorithms for Wireless, Mobile, Ad Hoc and Sensor Networks (WMAN) of IPDPS, 2004

[26] S. Zhong, L.E. Li, Y.G. Liu, and Y.R. Yang, "On designing incentivecompatible routing and forwarding protocols in wireless ad-hoc networks: an integrated approach using game theoretical and cryptographic techniques," in Proc. 11th ACM annual international conference on Mobile computing and networking (MobiCom), 2005, pp. 117-131.

[27] S. Zhong, J. Chen, and Y. Yang, "Sprite: A simple, cheatproof, creditbased system for mobile ad hoc networks," in Proc. 22nd IEEE Conference on Computer and Communications, (INFOCOM), 2003.

[28] M. Lu, F. Li, and J. Wu, "Incentive compatible cost- and stability-based routing in ad hoc networks," in Proc. 12th International Conference on Parallel and Distributed Systems (ICPADS), 2006, pp. 495-500.

[29] W. Wang, X.Y. Li, and Y. Wang, "Truthful multicast in selfish wireless networks," in Proc. 10th ACM annual international conference on Mobile computing and networking (MobiCom), 2004, pp. 245-259.

[30] N. Rama Suri and Y. Narahari, "Design of incentive compatible protocols for ad hoc wireless networks," Tech. Rep., Dept. of CSA, IISc, Bangalore., 2007.

[31] N. Rama Suri and Y. Narahari, "Broadcast in ad hoc wireless networks with selfish nodes: A bayesian incentive compatibility approach," in 2nd IEEE/Create-Net/ICST International Conference on COMmunication System softWAre and MiddlewaRE (COMSWARE), 2007.

[32] D. Garg, Design of Innovative Mechanisms for Contemporary Game Theoretic Problems in Electronic Commerce, Ph.D. thesis, Dept. of CSA, Indian Institute of Science, June, 2006.

[33] P. Maille and B. Tuffin, "Why VCG auctions can hardly be applied to the pricing of inter-domain and ad hoc networks," Tech. Rep., GET/ENST Bretagne, France, 2006.

[34] D. Garg, Y. Narahari, and S. Gujar, "Foundations of mechanism design: Part 1 - fundamental concepts and key results," Sadhana, Indian academy Proc. Engineering Sciences (http://lcm.csa.iisc.ernet.in/gametheory/md1-dec07.pdf), 2008.

[35] S. Zhong, L.E. Li, Y.R. Yang, and Y. Liu, "On designing incentivecompatible routing and forwarding protocols in wireless ad-hoc networks - an integrated approach using game theoretical and cryptographic techniques," ACM/Baltzer Wireless Networks (WINET), 2006.

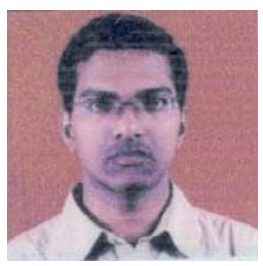

N. Rama Suri received M.Sc.(Engg.) degree in computer science, in 2006, from the Indian Institute of Science, Bangalore, India. He is currently a Ph.D. scholar in Indian Institute of Science, Bangalore, India. His current research interests include game theory, mechanism design, ad hoc wireless networks, social networks, electronic commerce, WWW. He is recipient of Microsoft Research India Ph.D fellowship.

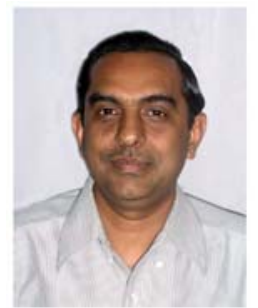

Dr. Y. Narahari is currently a Professor at the Department of Computer Science and Automation, Indian Institute of Science, Bangalore. He completed his Ph.D. at the same Department in 1988, with his Doctoral Dissertation on Petri Nets winning the Best Thesis Award for Electrical Sciences at the Indian Institute of Science. His current research focuses on the use of game theory and mechanism design in network economics problems. He is currently completing a research monograph entitled Emerging Game Theoretic Problems in Network Economics and Mechanism Design Solutions, to be published by Springer, London. $\mathrm{He}$ has spent sabbaticals at the Massachusetts Institute of Technology, Cambridge, Mass, USA, in 1992 and at the National Institute of Standards and Technology, Gaithersburg, MD, USA, in 1997. He is currently on the editorial board of IEEE Transactions on Systems, Man \& Cybernetics (Part A) and IEEE Transactions on Automation Science \& Engineering (where he is a Senior Editor). Dr. Narahari is a Fellow of the IEEE, a fellow of the Indian National Academy of Engineering, a fellow of the Indian National Academy of Sciences, and a Homi Bhabha Research Fellow. He has been involved in several high impact collaborative research projects with General Motors R \& D, Intel, and Infosys Technologies. 